\title{
Two Simple Families of Exact Inhomogeneous Stiff Cosmologies
}

\author{
Guillermo A. González, Fabio D. Lora, and Jenrry A. Jaimes \\ Grupo de Investigación en Relatividad y Gravitación \\ Escuela de Física, Universidad Industrial de Santander \\ A.A. 678, Bucaramanga, Colombia \\ Received on 17 July, 2007
}

\begin{abstract}
Two families of exact simple solutions of Einstein field equations for inhomogeneous stiff cosmologies are presented. The method to obtain the solutions is based on the introduction of auxiliary functions in order to cast the Einstein equations in such a way that can be explicitly integrated. Although the equations are mathematically equivalent to the equations obtained when the source of matter is a scalar field, it is worth to mention that the source that we consider is not a scalar field but a perfect fluid with a stiff equation of state. The obtained solutions are expressed in terms of simple functions of the used coordinates and two families of particular solutions are considered. The geometrical and kinematical properties of the solutions are then analyzed and the parameters are restricted in order to have a physically acceptable behavior. The two particular solutions are of the Petrov type I, the first one being regular everywhere whereas the second one presents a big-bang singularity. Now, for a particular value of one of the parameters, the second particular solution is a vacuum solution of the Bianchi I type that reduces to the Kasner solution.
\end{abstract}

Keywords: Exact solutions; Cosmology; General Relativity

\section{INTRODUCTION}

The main motivation for the formulation of the standard physical cosmology, in which the universe is described as isotropic and completely homogeneous in all its evolution[14], has been the observable fact that the present universe seems to be isotropic and spatially homogeneous. However, as it is shown by a series of recent observations [5-7], our universe certainly is neither exactly homogeneous nor isotropic, and also there is not sufficient reason to believe that the behavior of its expantion was regular at early times. Thus, in order to understand the evolution and large-scale structure of the universe, it is necessary to consider a more general class of cosmologies obtained by removing the requirement of homogeneity and isotropy. Accordingly with the above considerations, in the last two decades there has been an increasing interest in the study of anisotropic and inhomogeneous cosmologies, as can be see, for instance, in the references [813]. (See also the reviews[14-19] for a summary of the main work).

Now, between the several methods used to study the spatially inhomogeneous cosmologies, the search for exact solutions of Einstein field equations plays a specially important role. Indeed, due to the highly nonlinear character of the field equations, the knowledge of some exact solutions is crucial for the understanding of specific qualitative aspects that can be used as a guide for the study of more general models. Now then, the properties of the exact solutions must be related and compared with the results obtained by other different methods, such as the methods of dynamical systems[20], the methods of the theory of perturbations[21] or the study of the structure and formation of singularities[22].

However, due to the mathematical complexity of the generic inhomogeneous models, the studies of exact inhomogeneous cosmologies have been limited mainly to the case of spacetimes admitting an Abelian two-parameter group of isometries, $G_{2}$, and were initiated with the study of Gowdy of spatially compact models[23, 24]. Now, if the metric admits an orthogonally transitive two-parameter group of isometries, then it can be written in a blocks diagonal form[21]. This kind of metrics admits two spacelike commuting Killing vector fields and, as can be easily verified[25], the corresponding vacuum Einstein equations leads to a complete integrable system of partial differential equations.

On the other hand, when we consider the non vacuum Einstein equations, the mathematical complexity makes it very difficult to find solutions with reasonably realistic fluids. So, in almost all the works on exact inhomogeneous cosmologies, the solutions are obtained by taking fluids whose equation of state is of the form $p=\gamma \rho$ and, in particular, with $\gamma=1$. This last case, the stiff fluid state equation, leads to a complete integrable system of partial differential equations and was considered by Zeldovich[26] as a good candidate in order to describe the matter content of the Universe in its earlier stage.

In agreement with the above considerations, in the present paper we present two families of simple exact inhomogeneous stiff cosmologies. The method to obtain the solutions is based on the introduction of auxiliary functions in order to cast the Einstein equations in such a way that can be explicitly integrated and the obtained solutions can be expressed in terms of simple functions of the used coordinates. Now, it is worth to mention that the equations are written in a way that are mathematically equivalent to the equations obtained when the source of matter is a scalar field. However, in spite of this mathematical equivalence, the source that we are considering is a perfect fluid with the stiff equation of state and not a scalar field.

The paper is organized as follows. First, in Sec. II, we present the Einstein and matter evolution equations and the integration procedure to obtain an explicit general solution. Then, in Sec. III, we consider two families of simple particular solutions and analyze their geometrical and kinematical properties. Finally, in Sec. IV, we summarize our main results. 


\section{THE EINSTEIN AND MATTER EVOLUTION EQUATIONS}

In order to study inhomogeneous stiff cosmologies, we take as the starting point the metric tensor as given by the line element[21]

$$
d s^{2}=e^{-2 U}\left[e^{2 \gamma}\left(-d t^{2}+d r^{2}\right)+W^{2} d x^{2}\right]+e^{2 U} d y^{2},
$$

where $U, \gamma$ and $W$ are functions of $r$ and $t$ only. We also consider as the matter contents a perfect fluid with the stiff equation of state $p=\rho$, whose energy-momentum tensor can be written as

$$
T_{\alpha \beta}=\rho\left(2 u_{\alpha} u_{\beta}+g_{\alpha \beta}\right) .
$$

With the above choices, the Einstein equations can be cast as

$$
R_{\alpha \beta}=2 \rho u_{\alpha} u_{\beta},
$$

whereas the matter evolution equations can be obtained, from the conservation law

$$
T_{; \beta}^{\alpha \beta}=0,
$$

by projecting it along the temporal and spatial directions. In order to obtain the above projections, we contract the equation (4) with the velocity vector $u^{\alpha}$ and the "spatial projection tensor" $h_{\alpha \beta}=u_{\alpha} u_{\beta}+g_{\alpha \beta}$, respectively. So we obtain

$$
\begin{aligned}
& \rho_{, \beta} u^{\beta}+2 \rho u_{; \beta}^{\beta}=0, \\
& 2 \rho u^{\beta} u_{; \beta}^{\alpha} h_{\alpha}^{\mu}+\rho_{, \beta} g^{\beta \alpha} h_{\alpha}^{\mu}=0,
\end{aligned}
$$

where we have used the condition $u_{\alpha} h^{\alpha \beta}=0$.
We now impose the irrotationality condition [27]

$$
u_{\alpha}=\frac{\Phi_{, \alpha}}{\left(-\Phi_{, \mu} \Phi^{, \mu}\right)^{1 / 2}}
$$

so that the equation (6) can be cast as

$$
\frac{\rho\left(\Phi_{, \mu} \Phi^{, \mu}\right)_{, \alpha} h_{\mu}^{\alpha}}{\left(\Phi_{, \mu} \Phi^{, \mu}\right)}=\rho_{, \alpha} h_{\mu}^{\alpha}
$$

which can be identically satisfied if we choose [28]

$$
\rho=-\frac{F}{2} \Phi_{, \mu} \Phi^{\mu}
$$

where $F$ is an arbitrary function of the scalar potential $\Phi$. Now, by using (9), the energy-momentum tensor can be cast as

$$
T_{\alpha \beta}=F\left[\Phi_{, \alpha} \Phi_{, \beta}-\frac{1}{2} g_{\alpha \beta} \Phi_{, \mu} \Phi^{, \mu}\right]
$$

in such a way that the Einstein and evolution equations can be written as

$$
\begin{gathered}
R_{\alpha \beta}=F \Phi_{, \alpha} \Phi_{, \beta}, \\
F \Phi_{; \alpha}^{, \alpha}=-\frac{F^{\prime}}{2} \Phi_{, \mu} \Phi^{, \mu},
\end{gathered}
$$

where $F^{\prime}=\frac{\partial F}{\partial \Phi}$.

For the metric (1), the equation (12) reduces to

$$
\Phi_{, r r}-\Phi_{, t t}+W^{-1}\left(W_{, r} \Phi_{, r}-W_{, t} \Phi_{, t}\right)+\frac{F^{\prime}}{2 F}\left(\Phi_{, r}^{2}-\Phi_{, t}^{2}\right)=0 .
$$

In order to solve the above equation, we assume that $\Phi=\Phi(\psi)$, where $\psi$ is a new scalar potential, in such a way that (13) can be written as

$$
\psi_{, r r}-\psi_{, t t}+W^{-1}\left(W_{, r} \psi_{, r}-W_{, t} \psi_{, t}\right)+\left[\frac{\Phi^{\prime \prime}}{\Phi^{\prime}}+\frac{F^{\prime} \Phi^{\prime}}{2 F}\right]\left(\psi_{, r}^{2}-\psi_{, t}^{2}\right)=0
$$

Now, we choose the functional dependence of $\Phi(\psi)$ in such a way that the above equation can be linearized. In order to do this, we take the expression in the square brackets as equal to zero,

$$
\frac{\Phi^{\prime \prime}}{\Phi^{\prime}}+\frac{F^{\prime} \Phi^{\prime}}{2 F}=0
$$

in such a way that

$$
k \psi=\int \sqrt{F} d \Phi
$$

where $k$ is an arbitrary positive constant.

Now, by using (16), is easy to see that, for any arbitrary function $F(\Phi)$, the energy density $\rho$, the velocity vector $u_{\alpha}$, the energy-momentum $T_{\alpha \beta}$ end the Einstein system of equa- 
tions can be cast as

$$
\begin{aligned}
& \rho=-\frac{k^{2}}{2} \psi_{, \mu} \psi^{, \mu}, \\
& u_{\alpha}=\frac{\psi_{, \alpha}}{\sqrt{-\psi_{, \mu} \psi^{, \mu}}}, \\
& T_{\alpha \beta}=k^{2}\left\{\psi_{, \alpha} \psi_{, \beta}-\frac{1}{2} g_{\alpha \beta} \psi_{, \mu} \psi^{\mu}\right\}, \\
& R_{\alpha \beta}=k^{2} \psi_{, \alpha} \psi_{, \beta} .
\end{aligned}
$$

As we can see, if we take $k=0$, the above system of equations reduce to the Einstein vacuum equations. Also, it is worth to mention that the equations are written in a way that are mathematically equivalent to the equations obtained when the source of matter is a scalar field. However, in spite of this mathematical equivalence, the source that we are considering is a perfect fluid with the stiff equation of state and not a scalar field.

By using the non vanishing components of the Ricci tensor for the metric (1), the Einstein and evolution equations can be written as the following system of partial differential equations

$$
\begin{aligned}
& W_{, r r}-W_{, t t}=0 \\
& \left(W \psi_{, r}\right)_{, r}-\left(W \Psi_{, t}\right)_{, t}=0 \\
& \left(W U_{, r}\right)_{, r}-\left(W U_{, t}\right)_{, t}=0, \\
& \gamma_{, t} W_{, r}+\gamma_{, r} W_{, t}=2 W U_{, t} U_{, r}+k^{2} W \psi_{, t} \psi_{, r}+w_{, t r}, \\
& \gamma_{, t} W_{, t}+\gamma_{, r} W_{, r}=W\left(U_{, t}^{2}+U_{, r}^{2}\right)+\frac{1}{2}\left[k^{2} W\left(\psi_{, t}^{2}+\psi_{, r}^{2}\right)+\left(W_{, t t}+W_{, r r}\right)\right] .
\end{aligned}
$$

As we can see, equation (21) is the classical one-dimensional wave equation, whose solutions are well known. On the other hand, from equations (22) and (23) we can see that $U(t, r)$ and $\psi(t, r)$ both are solutions of the same partial differential equation. So, in order to simplify the above system of equations, we can take $U(t, r)=\psi(t, r)$. Finally, the integrability conditions of the overdetermined system (24) - (25) are equivalent to the equations (21) - (23), guarantying so the existence of solutions. Now, as we can see from the above system of equations, the stiff fluids equations are easy to integrate due to the fact that, as a consequence of the stiff equation of state $p=\rho$, the equations for the metric functions $U$ and $W$ decouple from the pressure [29].

In order to solve the system (21) - (25), we first consider solutions of the equation (21) of the general form

$$
W(r, t)=\Psi(r+t)+\Omega(r-t),
$$

where $\Psi$ and $\Omega$ are arbitrary functions. We also consider another, linearly independent, solution of (21) written as

$$
V(r, t)=\Psi(r+t)-\Omega(r-t) .
$$

Now, by using $V(r, t)$ and $W(r, t)$, we define a coordinate transformation

$$
(t, r, x, y) \leftrightarrow(V, W, x, y),
$$

in such a way that the line element takes the form

$$
d s^{2}=e^{-2 U}\left[e^{2 \Lambda}\left(-d V^{2}+d W^{2}\right)+W^{2} d x^{2}\right]+e^{2 U} d y^{2},
$$

where $\Lambda$ is given by

$$
\Lambda=\gamma-\frac{1}{2} \ln \left(W_{, r}^{2}-W_{, t}^{2}\right) .
$$

The above coordinate transformation leads the Einstein and evolution equations to the form

$$
\begin{aligned}
& \Lambda, W=q W\left(\psi_{, V}^{2}+\psi_{, W}^{2}\right), \\
& \Lambda, V=2 q W \psi \psi_{, V} \psi_{, W}, \\
& \left(W \psi_{, V}\right)_{, V}-\left(W \psi_{, W}\right)_{, W}=0,
\end{aligned}
$$

where $q=1+k^{2} / 2$. As we can see, equation (33) is equivalent to the integrability condition of the overdetermined system (31) - (32).

We will now consider some simple solutions of the above system. In order to do this, we seek for solutions of (33) of the form $\psi(W, V)=A(W)+B(V)$ and obtain

$$
\psi=\frac{a_{1}}{4}\left(W^{2}+2 V^{2}\right)+a_{2} \ln W+a_{3} V,
$$

where $a_{1}, a_{2}$ and $a_{3}$ are real arbitrary constants. According with this, the solution of (31) - (32) is given by

$$
\begin{aligned}
\Lambda= & \frac{q}{2}\left(a_{1}^{2} V^{2}+a_{3}^{2}+2 a_{1} a_{3} V+a_{1} a_{2}\right) W^{2} \\
& +\frac{q a_{1}^{2}}{16} W^{4}+q a_{2}^{2} \ln W+q a_{1} a_{2} V^{2}+2 q a_{2} a_{3} V .
\end{aligned}
$$


Now, by choosing some particular solutions of (26) - (27), we can obtain many different families of solutions for the full system (21) - (25). So, in the next section, we will present two simple families of solutions obtained by means of a particularly simple form of the functions $\Psi$ and $\Omega$.

\section{TWO SIMPLE FAMILIES OF PARTICULAR SOLUTIONS}

In order to obtain simple particular solutions, we will consider the two simple functions

$$
\begin{aligned}
& f_{1}(r, t)=\frac{r+t}{2}, \\
& f_{2}(r, t)=\frac{r-t}{2},
\end{aligned}
$$

and we will take $\Psi$ and $\Omega$ as defined by

$$
\begin{aligned}
& \Psi(r, t)=f_{1}(r, t), \\
& \Omega(r, t)=f_{2}(r, t),
\end{aligned}
$$

for the first family of solutions, and

$$
\begin{aligned}
& \Psi(r, t)=f_{1}(r, t), \\
& \Omega(r, t)=-f_{2}(r, t),
\end{aligned}
$$

for the second family of solutions.

\section{A. The first family of solutions}

By taking the first family of solutions, we obtain for the metric functions the expressions

$$
\begin{aligned}
W(r, t)= & r, \\
\gamma(r, t)= & \frac{q}{2}\left(a_{1}^{2} t^{2}+a_{3}^{2}+2 a_{1} a_{3} t+a_{1} a_{2}\right) r^{2} \\
& +q \frac{a_{1}^{2}}{16} r^{4}+q a_{2}^{2} \ln r+q a_{1} a_{2} t^{2}+2 q a_{2} a_{3} t, \\
U(r, t)= & \frac{a_{1}}{4}\left(r^{2}+2 t^{2}\right)+a_{2} \ln r+a_{3} t,
\end{aligned}
$$

whereas for the fluid density we obtain the expression

$$
\rho=\frac{k^{2}}{2}\left[a_{1}\left(a_{1} t^{2}+2 a_{3} t\right)+a_{3}^{2}-\frac{a_{1}^{2} r^{2}}{4}-\frac{a_{2}^{2}}{r^{2}}-a_{1} a_{2}\right] e^{2(U-\gamma)},
$$

and for the velocity components the expressions

$$
\begin{aligned}
& u_{t}=\frac{k}{\sqrt{2 \rho}}\left(a_{1} t+a_{3}\right), \\
& u_{r}=\frac{k}{\sqrt{2 \rho}}\left(\frac{a_{1} r}{2}+\frac{a_{2}}{r}\right) .
\end{aligned}
$$

Now, in order to obtain a physically acceptable distribution of matter, we require that $\rho \geq 0$ for any value of $r$ and $t$. So, from the expression (45), is easy to see that $\rho$ will be no negative everywhere only if we take $a_{1}=a_{2}=0$. On the other hand, the requirement that the velocity vector be future oriented for any value of $r$ and $t$ imply that we must take $a_{3}<0$.

By imposing the above conditions, the line element (1) takes the following form

$$
d s^{2}=e^{-2 a_{3} t}\left[e^{q a_{3}^{2} r^{2}}\left(-d t^{2}+d r^{2}\right)+r^{2} d x^{2}\right]+e^{2 a_{3} t} d y^{2},
$$

so that the density is given by the expression

$$
\rho=\frac{k^{2} a_{3}^{2}}{2} e^{a_{3}\left(2 t-a_{3} q r^{2}\right)}
$$

whereas the fluid velocity takes the form

$$
u^{\alpha}=e^{a_{3}\left(2 t-a_{3} q r^{2}\right) / 2}(1,0,0,0)
$$

guarantying so the comoving nature of the reference frame.

Now, in order to see if the solution has any curvature singularity, we compute the components of the Weyl tensor in the natural null tetrad of the metric $[25,30]$, which are given by

$$
\begin{aligned}
& \Psi_{0}(t, r)=\frac{1}{2} a_{3}^{2}\left(2 a_{3} q r-q-2\right) e^{a_{3}\left(2 t-a_{3} q r^{2}\right)}, \\
& \Psi_{2}(t, r)=-\frac{1}{2} a_{3}^{2} e^{a_{3}\left(2 t-a_{3} q r^{2}\right)}, \\
& \Psi_{4}(t, r)=-\frac{1}{2} a_{3}^{2}\left(2 a_{3} q r+q+2\right) e^{a_{3}\left(2 t-a_{3} q r^{2}\right)},
\end{aligned}
$$

so that all of them are regular everywhere. Furthermore, is easy to see that the Weyl tensor is of Petrov type I.

The kinematical quantities of the metric can be also easily computed. Thus we obtain the following expressions for the acceleration, the expansion and the shear of the fluid

$$
\begin{aligned}
a_{\alpha} & =a_{3}^{2} q r(0,1,0,0), \\
\theta & =\left|a_{3}\right| e^{-\left(\left|a_{3}\right| t+a_{3}^{2} q r / 2\right),} \\
\sigma_{11} & =\frac{2\left|a_{3}\right|}{3} e^{\frac{a_{3}}{2}\left(a_{3} q r^{2}-2 t\right),} \\
\sigma_{22} & =\frac{2\left|a_{3}\right|}{3} r^{2} e^{-\frac{a_{3}}{2}\left(a_{3} q r^{2}+2 t\right),} \\
\sigma_{33} & =-\frac{4\left|a_{3}\right|}{3} r^{2} e^{-\frac{a_{3}}{2}\left(a_{3} q r^{2}-6 t\right)},
\end{aligned}
$$

where all the components have been computed in the natural orthonormal tetrad of the metric. As we can see the kinematical quantities are regular everywhere. Also is easy to see that, as $a_{3}<0$, the components $\sigma_{11}$ and $\sigma_{22}$ are positive, while $\sigma_{33}$ is negative. 


\section{B. The second family of solutions}

Now, by taking the second family of solutions, we obtain for the metric functions the expressions

$$
\begin{aligned}
W(r, t)= & t \\
\gamma(r, t)= & q\left(a_{1}^{2} r^{2}+a_{3}^{2}+2 a_{1} a_{3} r+a_{1} a_{2}\right) \frac{t^{2}}{2} \\
& +q \frac{a_{1}^{2}}{16} t^{4}+q a_{2}^{2} \ln t+q a_{1} a_{2} r^{2}+2 q a_{2} a_{3} r, \\
U(r, t)= & \frac{a_{1}}{4}\left(t^{2}+2 r^{2}\right)+a_{2} \ln t+a_{3} r
\end{aligned}
$$

in such a way that the fluid density is given by

$$
\rho=\frac{k^{2}}{2}\left[\frac{a_{1}^{2} t^{2}}{4}+\frac{a_{2}^{2}}{t^{2}}+a_{1} a_{2}-a_{1}\left(a_{1} r^{2}+2 a_{3} r\right)-a_{3}^{2}\right] e^{2(U-\gamma)},
$$

whereas the velocity components are given by

$$
\begin{aligned}
& u_{r}=\frac{k}{\sqrt{2 \rho}}\left(a_{1} r+a_{3}\right), \\
& u_{t}=\frac{k}{\sqrt{2 \rho}}\left(\frac{a_{1} t}{2}+\frac{a_{2}}{t}\right) .
\end{aligned}
$$

Now, as in the first family of solutions, we require that $\rho \geq 0$ for any value of $r$ and $t$ in order to obtain a physically acceptable distribution of matter. From expression (62) is easy to see that $\rho$ will be no negative everywhere only if we take $a_{1}=a_{3}=0$, so that the expression for the density reduces to

$$
\rho=\frac{k^{2} a_{2}^{2}}{2} t^{-2\left(q a_{2}^{2}-a_{2}+1\right)}
$$

Now, as $\left(a_{2}-1\right) /\left(a_{2}^{2}\right)<1<q$, we have an initial singularity and then the density decreases to zero as $t \rightarrow \infty$. On the other hand, the velocity vector is given by

$$
u^{\alpha}=t^{a_{2}\left(1-q a_{2}\right)}(1,0,0,0),
$$

where, in order to have a future oriented timelike vector, we have taken $a_{2}<0$. Also, we can see that the spatial velocity is zero and thus we again have a comoving reference frame.

The line element can be written as follows

$$
d s^{2}=t^{-2 a_{2}}\left[t^{2 q a_{2}^{2}}\left(-d t^{2}+d r^{2}\right)+t^{2} d x^{2}\right]+t^{2 a_{2}} d y^{2},
$$

so that, when $q=1$ (or $k=0$ ) we have a vacuum solution of the Bianchi I type that reduces to the Kasner solution [31, 32], which can be written as $[21,33]$

$$
d s^{2}=t^{\left(d^{2}-1\right) / 2}\left(-d t^{2}+d r^{2}\right)+t^{1+d} d x^{2}+t^{1-d} d y^{2},
$$

with the Kasner parameter given by $d=1-2 a_{2}$. Now, it is worth to mention that another kind o inhomogeneous stiff cosmologies were obtained by Patel and Dadich [34] which also reduce to the Kasner solution. However, in contrast with the solution here presented, the solutions of Patel and Dadich are singularity free.
Now, in order to see if the solution has a real initial singularity, we computed the Weyl tensor in the natural null tetrad of the metric $[25,30]$ and obtain

$$
\begin{aligned}
& \Psi_{0}(t, r)=\frac{1}{2} a_{2}\left(2 a_{2}-1\right)\left(a_{2} q-1\right) t^{-2 q a_{2}^{2}+2 a_{2}-2}, \\
& \Psi_{2}(t, r)=-\frac{1}{2}\left(a_{2}-1\right) a_{2} t^{-2 q a_{2}^{2}+2 a_{2}-2}, \\
& \Psi_{4}(t, r)=\frac{1}{2} a_{2}\left(2 a_{2}-1\right)\left(a_{2} q-1\right) t^{-2 q a_{2}^{2}+2 a_{2}-2} .
\end{aligned}
$$

The scalars constructed from the Ricci and Weyl tensors diverge as $t \longrightarrow 0$, which corresponds to a big-bang singularity. Also, it is easy to see that in the algebraic classification of the Reimann tensor, the metric is of Petrov type I.

On the other hand, the kinematical quantities for this model can also be easily computed and so, by taking $a_{2}<0$, we obtain for the non-vanishing components of the acceleration, expansion and shear of the fluid the following expressions

$$
\begin{aligned}
a_{\alpha} & =(0,0,0,0) \\
\theta & =\frac{\left(a_{2}^{2} q-a_{2}+1\right)}{t^{\left(a_{2}^{2} q-a_{2}+1\right)}} \\
\sigma_{11} & =\frac{\left(2 a_{2}^{2} q-2 a_{2}-1\right) t^{\left(a_{2}^{2} q-a_{2}-1\right)}}{3} \\
\sigma_{22} & =-\frac{\left(a_{2}^{2} q+2 a_{2}-2\right) t^{-\left(a_{2}^{2} q+a_{2}-1\right)}}{3} \\
\sigma_{33} & =-\frac{\left(a_{2}^{2} q-4 a_{2}+1\right) t^{-\left(a_{2}^{2} q-3 a_{2}+1\right)}}{3}
\end{aligned}
$$

where all the components have been computed in the natural orthonormal tetrad of the metric. It is interesting to see that, as the pressure gradient is zero, the acceleration is equal to zero and thus the fluid is geodesic. On the other hand, as $a_{2}<0$, all the non-vanishing components of the shear are negative.

\section{DISCUSSION}

We presented two simple families of exact inhomogeneous stiff cosmologies. The solutions were obtained by explicitly integrating the Einstein and matter evolution equations by means of the introduction of an auxiliary function that leads to a complete integrable system of partial differential equations. Now, it is worth to mention that the equations were written in a way that are mathematically equivalent to the equations obtained when the source of matter is a scalar field. However, despite of this mathematical equivalence, the source that we consider was not a scalar field but a perfect fluid with the stiff equation of state.

A general solution was obtained that can be expressed in terms of simple functions of the used coordinates. Then, two families of particular solutions were considered, and their geometrical and kinematical properties were analyzed and the values of the parameters were restricted in order to have a physically acceptable behavior. The two particular solutions are 
of the Petrov type I, the first one being regular everywhere whereas the second one presents a big-bang singularity.

Now, for a particular value of one of the parameters, the second particular solution is a vacuum solution of the Bianchi I type that reduces to the Kasner solution. However, although there are some other exact inhomogeneous stiff cosmologies that in the vacuum reduce to the Kasner solution, as it is the case with the solutions obtained by Patel and Dadich [34], it is worth to mention that this is not a characteristic behavior of this kind of cosmologies. Indeed, an example of a different behavior is shown by the first particular solution considered in this paper, which in the vacuum is not of the Bianchi I type and do not reduces to the kasner solution.

\section{Acknowledgments}

F. D. Lora wants to thank the finantial support from Vicerrectoría Académica, Universidad Industrial de Santander.
[1] P. J. E. Peebles, Physical Cosmology (Princeton University Press, 1971).

[2] P. J. E. Peebles, Principles of Physical Cosmology (Princeton University Press, 1993).

[3] S. Weinberg, Gravitation and Cosmology (John Wiley, 1972).

[4] Ya. B. Zeldovich and I. D. Novikov, Relativistic Astrophysics: The structure and evolution of the Universe (University of Chicago Press, 1983).

[5] G. F. Smoot et al., Astrophys. J. 396, L1 (1992).

[6] C. L. Bennet et al., Astrophys. J. Suppl. Ser. 148, 1 (2003).

[7] D. N. Spergel et al., Astrophys. J. Suppl. Ser. 148, 175 (2003).

[8] J. Wainwright and B. J. Marshman, Phys. Lett. 72A, 275 (1979).

[9] J. Wainwright, Phys. Rev.D 20, 3031 (1979).

[10] J. Wainwright, J. Phys. A 12, 2015 (1979).

[11] M. Carmeli and Ch. Charac, Phys. Lett. A 75, 333 (1980).

[12] J. Wainwright, J. Phys. A 14, 1131 (1981)

[13] A. D. Adams, R. W. Hellings, R. L. Zimmerman, H. Farhoosh, D. I. Levine, and Z. Zeldich, Astrophys. J. 253, 1 (1982).

[14] M. Carmeli, Ch. Charac, and S. Malin, Phys. Rep. 76, 79 (1981).

[15] W. B. Bonnor, J. B. Griffiths, and M. A. H. McCallum, Gen. Rel. Grav. 26, 687 (1994).

[16] M. A. McCallum, in Lecture Notes in Physics: Retzbach Seminar on Exact Solutions of Einstein's field Equations, eds. W. Dietz and C- Hoenselaers (Springer Verlag, 1984).

[17] M. A. MacCallum, in The origin of structure in the universe, eds. E. Gunzig and P. Nardone (Kluwer Academic, 1993).

[18] M. A. MacCallum, in The renaissance of general relativity and cosmology, eds. G. F. R. Ellis, A. Lanza and J. C. Miller (Cambridge University Press, 1993).

[19] E. Verdaguer, Phys. Rep. 229, 1 (1993).

[20] J. Wainwright and G. F. R. Ellis, Eds. Dynamical Systems in Cosmology, (Cmabridge University Press, 1977).

[21] H. Stephani, D. Kramer, M. MacCallum, C. Hoenselaers, and E. Herlt, Exact solutions of Einstein's field equations (Cambridge University Press, 2003).

[22] J. M. M. Senovilla, Gen. Rela. Gravit. 30, 701 (1998).

[23] R. H. Gowdy, Phys. Rev. Lett. 27, 826 (1971).

[24] R. H. Gowdy, Ann. Phys. 83, 203 (1974).

[25] V. Belinski and E. Verdaguer, Gravitational Solitons (Cambridge University Press, 2001).

[26] Y. B. Zeldovich, Month. Not. Roy. Astron. Soc. 160, 1P, (1972).

[27] A. H. Taub, Comm. Math. Phys. 29, 79 (1973).

[28] P. S. Letelier, Phys. Rev. D 26, 2623 (1982).

[29] L. Fernández-Jambrina and L.M. González-Romero, J. Math. Phys. 45, 2113,(2004)

[30] A. Feinstein and J. M. M. Senovilla, Class. Quantum Grav. 6, L89 (1989).

[31] E. Kasner, Am. J. Math. 43, 217 (1921).

[32] L. D. Landau and E. M . Lifshitz, Théorie des Champs (Éditions MIR, Moscou, 1970).

[33] V. A. Belinski, I. M. Khalatnikov, and E. M. Lifshitz, Adv. Phys. 19, 525 (1970).

[34] L. K. Patel and N. Dadich, Singularity Free Inhomogeneous Cosmological Stiff Fluid Models, gr-qc/9302001 (1993). 